\title{
Plan de negocios: elaboración de toallas sanitarias y pañales para la incontinencia a base de fécula de maíz y con materiales biodegradables
}

\section{Business Plan: Elaboration of sanitary towels and dies for incontinence based on corn facture and with biodegradable materials}

Rosa Mercedes Zabala Cuadrado. ${ }^{1}$, Mersi Piedad Mantilla Jacome. ${ }^{2}$, Narcisa Elisabeth Saltos. ${ }^{3}$

DOI: https://doi.org/10.33262/visionariodigital.v3i3.771

\begin{abstract}
The use of feminine towels is almost mandatory for all women when they are in their menstrual period. A woman, throughout her fertile life, which is from 15 to 44 years, according to the World Health Organization (WHO), uses about 15,000 disposable towels. Main objective to know the product's consumption preferences towels sanitary and diapers ECOMAIZ, by the citizens of the city of Riobamba. Reach to be innovative in the development of the product thinking about the benefit of women in their menstrual period and having incontinence. Methodology made with corn starch and materials that degrade in 6 months, the surface of each diaper is covered by a film made of natural corn, $100 \%$ free of transgenic, which allows the skin of the elderly is dry is made in $70 \%$ with natural and biodegradable materials. Results in surveys carried out on people aged 12 and over, $74 \%$ of respondents know they are organic products, $94 \%$ want to acquire our product and $100 \%$ receive information about them, which helps us to be able to launch the product to the market with an economical price. Conclusion Sanitary towels and diapers for incontinence "ECOMAÍZ", are a very promising and innovative alternative, since these are those who have different vision of helping the environment.
\end{abstract}

Keywords: Incontinence, Innovation, Menstruation, Corn.

\footnotetext{
${ }^{1}$ Escuela Superior Politécnica de Chimborazo, Facultad de Ciencias, Riobamba, Ecuador. rosa.zabala@espoch.edu.ec

${ }^{2}$ ESPE. Departamento de Seguridad y Defensa, Chimborazo, Ecuador, mpmantilla@espe.edu.ec

${ }^{3}$ ESPE. Departamento de Seguridad y Defensa, Chimborazo, Ecuador, nesaltos1@espe.edu.ec
} 


\section{RESUMEN}

El uso de toallas femeninas es casi obligado para todas las mujeres cuando se encuentran en su periodo menstrual. Una mujer, a lo largo de su vida fértil, que es de los 15 a los 44 años, esto según la Organización Mundial de la Salud (OMS), utiliza alrededor de 15,000 toallas desechables. Objetivo principal conocer las preferencias de consumo del producto toallas sanitarias y pañales Ecomaiz, por parte de las ciudadanas /os de la cuidad de Riobamba. Alcance ser innovadores en la elaboración del producto pensando en el beneficio de las mujeres en su período menstrual y que presentan incontinencias. Metodologías elaboradas con fécula de maíz y con materiales que se degradan en 6 meses, la superficie de cada pañal está cubierta por una película hecha de maíz natural, 100\% libre de transgénicos, que permite que la piel de adulto mayor esté seca está elaborado en un $70 \%$ con materiales naturales y biodegradables. Resultados en las encuestas realizadas a personas de 12 años en adelante, se obtuvo $74 \%$ de los encuestados saben que son los productos ecológicos, un $94 \%$ desean adquirir nuestro producto y un $100 \%$ recibir información de los mismos, lo que nos ayuda para poder lanzar el producto al mercado con un precio económico. Conclusión las Toallas higiénicas y pañales para la incontinencia "ECOMAÍZ", son una alternativa muy prometedora e innovador, ya que estos son los que tienen visión diferente de ayudar al medio ambiente.

Palabras claves: incontinencia, innovación, menstruación, maíz

\section{Introducción.}

El surgimiento de la prensa femenina da un excelente soporte a la publicidad de este tipo de productos. Primero con la revista El Hogar (1904-1962) y luego, con la publicación de la que sería una de las publicaciones más importantes dentro de la prensa destinada a la mujer de la época, y aún de la actualidad, la revista Para Ti (1922) (Barros, 2009).

El uso de toallas femeninas es casi obligado para todas las mujeres cuando se encuentran en su periodo menstrual. Una mujer, a lo largo de su vida fértil, que es de los 15 a los 44 años, esto según la Organización Mundial de la Salud (OMS), utiliza alrededor de 15,000 toallas o tampones desechables (Salud, 2016).

Una mujer en promedio usara 17.000 toallas higiénicas en su vida fértil. Esos 17.000 productos higiénico llena de químicos, plásticos y otros compuestos sintéticos quedaran enterrados en los botaderos del mundo y tardaran mucho tiempo en descomponerse (Pessi, 2010).

Hoy en día, la oferta de productos para atender el ciclo menstrual es amplia y variada, a veces con fundamentos que superan el objetivo argumento de la rentabilidad económica y proponen formas de vida más sanas, libres, ecológicas, socialmente comprometidas, anti sistémicas y 
de empoderamiento (Tarzibachi, 2000). Así, para aliviar dolores y malestares, físicos y emocionales, podemos encontrar fármacos; pero también alternativas vinculadas con la ginecología natural y la medicina ancestral (Felitti, 2016).

Ante esta problemática de salud que enfrenta las mujeres se decidió crear toallas ecológicas, que sean cómodas en climas calurosos y no provoquen sudoración. Además, no generan el característico mal olor de las toallas desechables, optamos por este producto orgánico por que la tela y la fécula de maíz que las compone, se biodegradará en algunos meses, dependiendo de las condiciones del ambiente, excepto la capa impermeable

\section{Métodos Utilizados.}

El tipo de estudio realizado fue prospectivo, transverso y descriptivo, se llevó a cabo durante los meses de septiembre 2018 a febrero del 2019 y se lo realizó siguiente los pasos para preparar un Plan de negocios.

\section{Capítulo I Identificación del emprendimiento Descripción}

Sus diseños son llamativos, de diversos tamaños y colores y se sienten como ropa interior, no irritan o pican como las toallas desechables. Son cómodas en climas calurosos porque no provocan sudoración. Además, no generan el característico mal olor de las toallas desechables y contribuyen al ambiente por ser ecológicas y rápida degradación debido a su composición.

Estas poseen las siguientes características:

- Son hechas de fécula de maíz que además de ser impermeable son biodegradables.

- En cuanto a su diseño; se sujeta perfectamente a la ropa interior, lo que ofrece comodidad y seguridad.

- Contribuyen al ambiente porque son de rápida degradación en comparación con las toallas desechables que tardan años en degradarse.

\section{Competencia}

Nuestra principal competencia son las diferentes empresas que elaboran toallas sanitarias, que su principal componente con la que elaboran son sustancias químicas.

\section{Talentos}

Somos mujeres creativas y emprendedoras en llevar a cabo la elaboración de productos ecológicos que contribuyan al ambiente y a la comodidad femenina por las características que poseen. Además, son cien por ciento ecuatorianas favoreciendo a la economía del país.

\section{Ventaja competitiva}

No solamente se pensó en el beneficio de las personas sino también en realizar productos ecológicos amigables con el ambiente.

Solo basta con mirar a nuestro alrededor para darnos cuenta de que vivimos en la era de lo desechable, por esta razón se realizó productos ecológicos a base de fécula de maíz, donde 
el cultivo de maíz es sin duda, el más importante del país, el grano de maíz es el rubro más importante de las importaciones de productos agropecuarios del país.

\section{Modelo de negocios}

Va a comprender todos los elementos que permitan diseñar nuestro emprendimiento como tal, a través de la herramienta: CANVAS

\section{Capítulo II estudio de mercado} Segmentación del mercado

De acuerdo con nuestro producto se definió que:

- Por área demográfica: edad y sexo

- Uso del producto: frecuencia con la que se usa el producto

- Beneficio del producto: las necesidades que se cubrirán por el producto y la satisfacción de nuestros clientes.

\section{Proceso de estudio de mercado}

\section{Diseño de la investigación}

La fuente para nuestro diseño de investigación se tomó como base el promedio que utilizan toallas sanitarias las mujeres en edad fértil y donde realizan la adquisición de las mismas. Por lo tanto, se tomó como muestra cincuenta personas que serán cuestionadas en diferentes sitios de Riobamba.

\section{Técnica de recolección de información}

La técnica con la que se va recolectar información es mediante de las encuestas que se llevaron a cabo a diferentes personas de la ciudad de Riobamba. Para la distribución a efectuarse las encuestas se tomó una base como el mapeo de la ciudad y los sectores más concurridos.

\section{Recolección de información}

Se recolectó la información con la encuesta expuesta anteriormente, distribuyéndonos a diferentes sectores de la ciudad de Riobamba.

\section{Capítulo III plan de marketing Descripción}

Toallas sanitarias desechables y ecológicas para tu menstruación.

Ecológicas: Elaboradas con fécula de maíz y con materiales que se degradan en 6 meses. Necesitarás saber que las mujeres requieren estos productos desde la pubertad, que generalmente llega entre los 11 y 14. La superficie de cada pañal está cubierta por una película hecha de maíz natural, $100 \%$ libre de transgénicos, que permite que la piel de adulto mayor esté seca y que respire naturalmente.

Esta elaborado en un $70 \%$ con materiales naturales y biodegradables, lo que lo hace único frente a otros pañales desechables de prestaciones similares. 


\section{Características}

Presentaciones:

Toallas de día (10 toallas por paquete)

Nocturna (8 toallas por paquete)

\section{Forma de uso}

La recomendamos como alternativa a las mujeres que no quieren usar la copa menstrual y que no quieren seguir dañando el medio ambiente, ni estar en contacto con sustancias tóxicas que contienen las toallas sanitarias "ECOMAÍZ".

\section{Capítulo IV organización de la producción}

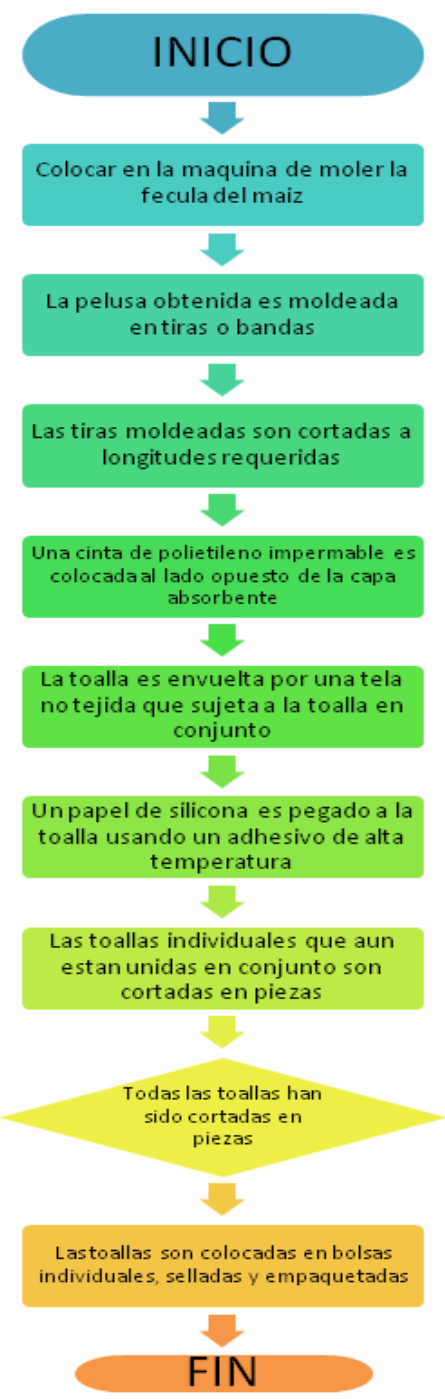

Figura 1. Flujograma de producción 


\section{Requerimientos}

Requerimientos de maquinaria y equipos

- Máquina de moler

- Compresor

- Cortador

- Maquina empacadora y selladora

\section{Requerimientos de recursos humanos}

- Operador

- Empacador

- Almacenador

- Supervisor

- Distribuidor

\section{Requerimientos de infraestructura}

- Área de recepción de materia prima e insumos

- Área de elaboración del producto

- Área de almacenamiento

- Área de análisis para el control de calidad

- Oficina

- Vestidor (para hombres y mujeres)

- Baños (para hombres y mujeres)

\section{Requerimientos de muebles y enseres}

- Equipos de bioseguridad (gorros, mandiles, gafas, guantes, botas, casco de protección, mascarilla, otros)

- Perchas

- Controladores de temperatura y humedad

- Ventiladores

- Materiales de limpieza

\section{Requerimientos de servicios}

- Agua

- Luz

- Internet

- Teléfono

\section{Requerimientos de materia prima e insumos}

- Fécula de maíz

- Adhesivo de alta temperatura 
- Telas no tejidas

- Papel fino

- Preservantes

\section{Capítulo V Estudio financiero}

Para realizar este estudio se utiliza información de varias fuentes, como estimaciones de ventas futuras, costos, inversiones a realizar, estudios de mercado, de demanda, costos laborales, costos de financiamiento, estructura impositiva, etc.

Se realiza todos estos procesos para analizar si nuestro emprendimiento y la inversión para nuestra empresa van a tener un flujo de caja positivo para nosotros.

\section{Resultados y Discusión}

\section{Resultados de Estudio Financiero}

La producción de toallas sanitarias y pañales para la incontinencia ECOMAIZ son de 4000 unidades diarias, esta cantidad se producirá debido al poco conocimiento de productos ecológicos.

Tabla 1. Inversiones

\begin{tabular}{lrrr}
\hline \multicolumn{1}{c}{ RUBROS } & INVERSIÓN & \multicolumn{1}{l}{ INVERSIÓN } & \multicolumn{1}{l}{ TOTAL } \\
& REALIZADA & POR REALIZAR & \\
\hline ACTIVOS FIJOS & & & \\
Terrenos & $20.000,00$ & 0,00 & $20.000,00$ \\
Edificaciones y construcciones & $11.500,00$ & 0,00 & $11.500,00$ \\
Vehículos & $30.000,00$ & 0,00 & \\
Maquinas, herramientas y equipos & $14.000,00$ & 0,00 & $14.000,00$ \\
Muebles y maquinaria de oficina & $8.330,00$ & 0,00 & $8.330,00$ \\
Equipos de cómputo y software & $2.500,00$ & 0,00 & $2.500,00$ \\
Subtotal & $\mathbf{8 6 . 3 3 0 , 0 0}$ & $\mathbf{0 , 0 0}$ & $\mathbf{5 6 . 3 3 0 , 0 0}$ \\
CAPITAL DE TRABAJO (3 MESES) & & & \\
Costos Materia prima (MP) & $5.120,00$ & 320,51 & $5.440,51$ \\
Costos Mano de obra (MO) & 527,20 & 527,20 & $1.054,40$ \\
Otros Costos & 6,15 & 6,15 & 12,30 \\
Subtotal & $\mathbf{5 . 6 5 3 , 3 5}$ & $\mathbf{8 5 3 , 8 6}$ & $\mathbf{6 . 5 0 7 , 2 1}$ \\
\hline INVERSIÓN TOTAL & $\mathbf{9 1 . 9 8 3 , 3 5}$ & $\mathbf{8 5 3 , 8 6}$ & $\mathbf{9 2 . 8 3 7 , 2 1}$ \\
\hline
\end{tabular}

Se realizará una inversión de $\$ 92.837,21$ estas separadas de acuerdo a las necesidades de la empresa, gastos de materia prima, muebles y enseres, infraestructura, materiales y equipo de bioseguridad, maquinaria y equipo entre otros. 
El costo de nuestras unidades a producir sea de $\$ 5,20$ cada paquete, por lo cual nuestra empresa generaría por el mismo precio toallas sanitarias nocturnas.

Tabla 2. Precio de venta

\begin{tabular}{|c|c|c|c|c|}
\hline DETALLE & $\begin{array}{c}\text { COSTO } \\
\text { UNITARIO \$ }\end{array}$ & \% UTILIDAD & $\begin{array}{c}\text { MARGEN DE } \\
\text { CONTRIBUCIÓN \$ }\end{array}$ & $\begin{array}{c}\text { PRECIO DE } \\
\text { VENTA por } \\
\text { unidad\$ }\end{array}$ \\
\hline $\begin{array}{c}\text { Toallas sanitarias y } \\
\text { pañales para } \\
\text { incontinencia }\end{array}$ & 5,20 & 4000 & & \\
\hline
\end{tabular}

Nuestro producto tuvo un costo por paquete de $\$ 5,20$ por lo que más la utilidad del $10 \%$ nos produjo un precio de venta de 5,72 por cada paquete más uno gratis nocturno.

Tabla 3. Flujo de Caja

\begin{tabular}{|c|c|c|c|c|c|c|c|}
\hline & & AÑO 0 & AÑO 1 & AÑO 2 & AÑO 3 & AÑO 4 & AÑO 5 \\
\hline+ & VENTAS NETAS & & $200.003,00$ & $203.123,00$ & $206.291,00$ & $209.510,00$ & $212.778,00$ \\
\hline- & COST. PRODUCC. & & $13.629,00$ & $14.041,00$ & $14.683,00$ & $15.583,00$ & $16.788,00$ \\
\hline- & GASTOS ADMINIS. & & $6.435,00$ & $6.629,00$ & $6.932,00$ & $7.357,00$ & $7.926,00$ \\
\hline- & GASTOS DE VENTAS & & $1.066,00$ & $1.098,00$ & $1.148,00$ & $1.219,00$ & $1.313,00$ \\
\hline- & COSTOS FINANCIEROS & & - & - & & - & \\
\hline$=$ & $\begin{array}{l}\text { UTILID. ANTES DE REPARTO DE } \\
\text { UTILIDADES E IMPUESTOS }\end{array}$ & & 178.873,00 & $181.355,00$ & $183.528,00$ & $185.351,00$ & $186.751,00$ \\
\hline- & REPARTO UTILIDADES (15\%) & & - & - & & - & \\
\hline$=$ & UTILIDADES ANTES DE IMPUESTOS & & 178.873,00 & $181.355,00$ & 183.528,00 & $185.351,00$ & 186.751,00 \\
\hline- & IMPUESTO A LA RENTA (25\%) & & - & - & - & - & \\
\hline$=$ & UTILIDAD NETA & & 178.873,00 & $181.355,00$ & $183.528,00$ & $185.351,00$ & $186.751,00$ \\
\hline- & $\begin{array}{l}\text { INVERSION EN MAQUINARIA Y } \\
\text { EQUIPO }\end{array}$ & -14000 & & & & & \\
\hline- & MUEBLES Y ENSERES & -8330 & & & & & \\
\hline- & INVERSIÓN TERRENO Y OBRA FISICA & -11500 & & & & & \\
\hline- & VEHICULO & & & & & & \\
\hline- & INV. CAPITAL DE TRABAJO (3 meses) & & $-15800,0$ & & & & \\
\hline+ & CAPITAL DE SOCIOS & & & & & & \\
\hline & FLUJO DE CAJA & -33830 & $163.073,00$ & $181.355,00$ & $183.528,00$ & $185.351,00$ & $186.751,00$ \\
\hline
\end{tabular}


TASA DE RENDIMIENTO DEL

MERCADO

$7,66 \%$

VAN: $\quad 639.298$

TIR:

El flujo de caja generado por nuestra empresa fue positivo ya que empezará a recuperar de las inversiones hechas en el primer año, dándonos un VAN de 639,298 y un TIR de 491\% por lo que nuestro producto es aceptado.

\section{Resultados del Estudio de Mercado}

Se realizó un total de 50 encuestas a los habitantes.

\section{Pregunta 1. ¿Es usted?}

Tabla 4. Sexo de los encuestados

\begin{tabular}{lcc} 
& Suma total & Porcentaje \\
\hline Mujer & 41 & 82 \\
Hombre & 9 & 18 \\
TOTAL & 50 & 100 \\
\hline
\end{tabular}

\section{Pregunta 2. Edad}

Tabla 5. Edad de los encuestados

\begin{tabular}{lcc}
\hline & $\begin{array}{c}\text { Suma } \\
\text { total }\end{array}$ & Porcentaje \\
\hline Entre 12 y 18 años & 4 & 8 \\
Entre 19 y 31 años & 21 & 42 \\
Entre 32 y 41 años & 12 & 24 \\
Entre 42 y 51 años & 6 & 12 \\
Entre 52 y 61 años & 4 & 8 \\
Entre 62 años en adelante & 3 & 6 \\
TOTAL & 50 & 100 \\
\hline
\end{tabular}

Pregunta 3. ¿Con qué frecuencia consume productos ecológicos?

Tabla 6. ¿Con qué frecuencia consume productos ecológicos?

\begin{tabular}{lcc} 
& Suma total & Porcentaje \\
\hline Diario & 4 & 8 \\
Una vez a la semana & 2 & 4 \\
Una vez al mes & 13 & 26 \\
Rara vez & 14 & 28 \\
No los consume & 17 & 34 \\
Otro & 0 & 0 \\
TOTAL & 50 & 100 \\
\hline
\end{tabular}


Pregunta 4. ¿Qué tipo de productos ecológicos ha consumido o le interesan?

Tabla 7. ¿Qué tipo de productos ecológicos ha consumido o le interesan?

\begin{tabular}{lcc}
\hline & Suma total & Porcentaje \\
\hline $\begin{array}{l}\text { Alimentos para requerimientos (veganos diabéticos, } \\
\text { celíacos...) }\end{array}$ & 3 & 6 \\
Desechables (papel, vasos, contenedores...) & 16 & 30 \\
Higiénicos (papel, servilletas, toallas de cocina...) & 17 & 32 \\
Cuidado personal y cosméticos & 15 & 28 \\
Otro & 2 & 4 \\
TOTAL & 53 & 100 \\
\hline
\end{tabular}

Pregunta 5. Si no sueles consumir productos ecológicos normalmente ¿Cuál es la causa?

Tabla 8. Si no sueles consumir productos ecológicos normalmente ¿Cuál es la causa?

\begin{tabular}{lcc}
\hline & Suma total & Porcentaje \\
\hline Son más caros & 13 & 26 \\
Son difíciles de encontrar & 31 & 62 \\
Son mejores los productos no naturales & 4 & 8 \\
Otro & 2 & 4 \\
TOTAL & 50 & 100 \\
\hline
\end{tabular}

Pregunta 6. ¿Qué precio estaría dispuesto a pagar por nuestros productos ecológicos

Tabla 9. ¿Qué precio estaría dispuesto a pagar por nuestros productos ecológicas

\begin{tabular}{lcc}
\hline & Suma total & Porcentaje \\
\hline 0,5 ctvs. & 30 & 60 \\
0,75 ctvs. & 13 & 26 \\
1,00 dólar & 7 & 14 \\
TOTAL & 50 & 100 \\
\hline
\end{tabular}

Pregunta 7. ¿Le gustaría recibir información recibir información y promociones sobre nuestros productos ecológicos?

Tabla 10. ¿Le gustaría recibir información recibir información y promociones sobre nuestros productos ecológicos?

\begin{tabular}{ccc}
\hline & Suma total & Porcentaje \\
\hline SI & 50 & 100 \\
NO & 0 & 0 \\
TOTAL & 50 & 100 \\
\hline
\end{tabular}


Es importante el uso de toallas sanitarias para el cuidado personal sin que estas puedan afectar la piel y causar irritaciones en las zonas íntimas de quienes las usan, por ello hemos diseñado un producto de uso intimo tanto para mujeres como para hombres (adultos mayores) también con el uso de este producto ayudaríamos al medio ambiente a que producir menos contaminación ya que nuestro producto tiene un tiempo de biodegradación menor a los otros productos ecológicos existentes en el mercado.

En las encuestas realizadas a personas de 12 años en adelante, tuvimos como resultado que un $74 \%$ de los encuestados saben que son los productos ecológicos, un 94\% desean adquirir nuestro producto y un $100 \%$ recibir información de los mismos, lo que nos ayuda para poder lanzar el producto al mercado con un precio económico.

Se pudo observar que el $34 \%$ de los encuestados no consumen productos ecológicos, ya que desconocen los beneficios que estos producen en el medio ambiente, el $28 \%$ rara vez los consume por falta de costumbre o de facilidad para obtenerlos y el $26 \%$ consumen estos productos una vez al mes ya que se les complica conseguirlos.

El 64\% de los encuestados prefieren que el producto se encuentre en una tienda, debido a que su acceso es más fácil y no requiere de mucho tiempo, mientras que el $20 \%$ de las personas indicaron que una buena forma de adquirir el producto es mediante un servicio a domicilio y con un $10 \%$ que el producto se expenda por medio del internet.

El $60 \%$ de las personas encuestadas optaron por el precio de 0.50 ctvs. observando de tal manera que las personas no miran el beneficio sino simplemente el costo, debido a que solo un $14 \%$ de los encuestados decidieron pagar $1 \$$, apreciando el tiempo y materia prima que se requiere producir toallas y pañales orgánicas

El total de los encuestados estuvieron completamente de acuerdo en recibir información y las promociones de los productos ecológicos que se van a producir, ya que a más información de los beneficios de nuestros productos la gente tendrá más conocimiento y de así podremos conseguir el aprecio por los productos a base de materia natural, también poder difundir a través del internet nuestro producto.

\section{CONCLUSIONES}

- Después de una investigación, se llegó a la conclusión de que las toallas higiénicas y pañales para la incontinencia "ECOMAÍZ", son una alternativa muy prometedora e innovador, ya que estos son los que tienen visión diferente de ayudar al medio ambiente y tendrá acogida por las personas debido a que no simplemente se producirá toallas sanitarias ecológicas sino también pañales para la satisfacción de los clientes. 
- Estos productos cumplirán con las expectativas y necesidades, y al ser un mercado poco explotado, se encuentra una gran oportunidad a la hora de satisfacerlos.

- Se contribuirá con nuestro producto al medio ambiente en vista a que es un producto de fácil degradación, amigable con el ambiente y que con una investigación de mercado y logrando educar al consumidor, se podrán lograr grandes cambios positivos para el ambiente.

- El estudio financiero realizado se demostró que sería viable en cuanto a la producción y los ingresos que se obtendrán de la misma, al tiempo de 5 años. De tal forma el beneficio es hacia los clientes, empresa y medio ambiente.

- Después de haber realizado el estudio financiero de nuestra empresa se obtuvo un VAN de 639.299 es decir que nuestro proyecto es aceptable, y una tasa interna de retorno de $491 \%$ es mayor a la que ofrece el mercado financiero, por ende, el producto es rentable.

\section{Referencias Bibliográficas}

Barros, C. (2009). Productos Ecológicos. Madrid: Visión Libros.

Bové, J. (2008). ¿Son caros los productos ecológicos? ¿Cuál es el verdadero precio de la comida? Vitónica, X (2). Chile: Editorial Planeta Chile.

Cajamar, I. (13 de enero de 2010). El mercado de productos ecológicos. Recuperado el 2 de noviembre de 2019, de El mercado de productos ecológicos: http://www.publicacionescajamar.es/pdf/series-tematicas/informes-coyunturamonografias/el-mercado-de-productos-ecologicos.pdf

Felitti, K. (2016). Sexualidad, Salud y Sociedad. IV (22). Obtenido de http://www.scielo.br/pdf/sess/n22/1984-6487-sess-22-00175.pdf

González Martínez, Á., Pérez Barrera, Y., \& Checa Godoy, A. (2014). El consumo de productos ecológicos en España. Marketing y Publicidad. España: Gremieditorscat.

Grant, D. (2000). El valor de los productos ecológicos. Ecogestos, III (4).

Guevara, J. (2018). La oferta de productos ecológicos aumenta. El Telégrafo, II (50).

Pessi, M. (2010). Análisis de la publicidad de productos para higiene femenina. II (12).

Pizarro, C. M. (13 de octubre de 2016). Consumo de Productos Ecológicos. Recuperado el 20 de febrero de 2019, de http://www.eduinnova.es/feb2010/ecologicos.pdf 
Vol. 3, N³., p.299 - 312, julio - septiembre, 2019

Pontanza, D. (30 de agosto de 2017). Toallas femeninas de tela ecológicas. Recuperado el 20 de febrero de 2019, de Toallas femeninas de tela ecológicas: https://tecreview.tec.mx/toallas-femeninas-tela-una-opcion-saludable-las-mujeres/

Quispe, J. A. (2014). La conciencia ecológica en el consumo de productos en la ciudad de puno Perú. $v(2)$.

Rivera, L. M. (16 de marzo de 2011). MARKETING DE PRODUCTOS ECOLÓGICOS. Recuperado el 20 de febrero de 2019, de MARKETING DE PRODUCTOS ECOLÓGICOS: $\quad$ http://www.publicacionescajamar.es/pdf/publicacionesperiodicas/mediterraneo-economico/3/3-26.pdf

Ruiz, M. (2013). Biodegradabilidad de Artículos Desechables en un Sistema de Composta. $X I V(2)$.

Salud, O. M. (18 de marzo de 2016). Datos Estadísticos OMS. Recuperado el 20 de febrero de 2019, de Datos Estadísticos OMS: https://www.who.int/gho/es/\#

Tarzibachi, E. (2000). Cosa de mujeres: Menstruación, género y poder. Argentina.

Xuesheng, Z. (16 de octubre de 2000). Fundamentos Guías de Estudio de Medicina China. Recuperado el 20 de febrero de 2019, de Fundamentos Guías de Estudio de Medicina China:

https://books.google.com.ec/books?id=ePTipPH1nNIC\&pg=PA125\&dq=EDAD+D

E+MUJER+CUANDO+EMPIEZA+LA+MENSTRUACI\%C3\%93N\&hl=es419\&sa=X\&ved=0ahUKEwi59MjN_czgAhVRhuAKHQBUBsAQ6AEINzAD\#v=0 nepage \&q=EDAD\%20DE\%20MUJER\%20CUANDO\%20EMPIEZA\%20LA\%20 MENSTRUACI\%C3\%93N\&f=false

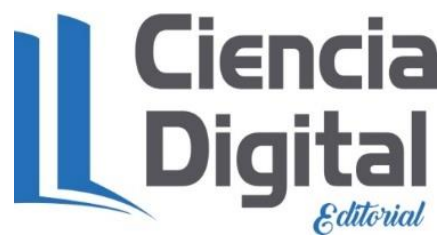




\section{PARA CITAR EL ARTÍCULO INDEXADO.}

Zabala Cuadrado, R., Mantilla Jacome, M., \& Saltos., N. (2019). Plan de negocios: elaboración de toallas sanitarias y pañales para la incontinencia a base de fécula de maíz y con materiales $\begin{array}{llll}\text { biodegradables. } \quad \text { Visionario } & \text { 299-312. }\end{array}$ https://doi.org/10.33262/visionariodigital.v3i3.771

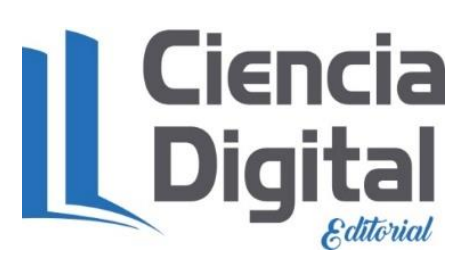

El artículo que se publica es de exclusiva responsabilidad de los autores y no necesariamente reflejan el pensamiento de la Revista Visionario Digital.

El artículo queda en propiedad de la revista y, por tanto, su publicación parcial y/o total en otro medio tiene que ser autorizado por el director de la Revista Visionario Digital.
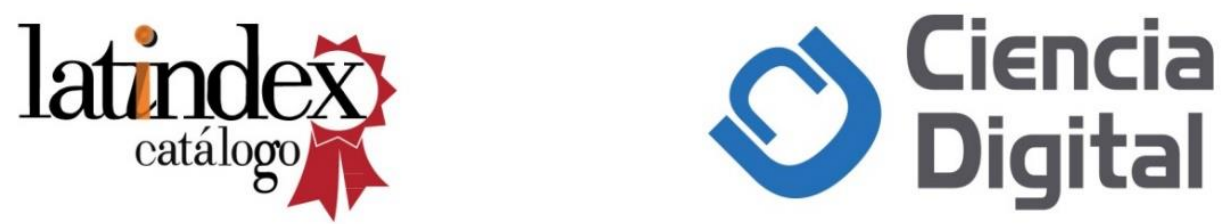